\title{
Epilepsy and Neurodevelopmental Outcomes in a Cohort of West Syndrome Beyond Two Years of Age
}

\author{
Reshma Aramanadka ${ }^{1}$ Jitendra Kumar Sahu ${ }^{2} \cdot$ Priyanka Madaan $^{2} \cdot$ Naveen Sankhyan $^{2} \cdot$ Prahbhjot Malhi $^{3}$. \\ Pratibha Singhi ${ }^{2,4}$ (1)
}

Received: 11 May 2021 / Accepted: 29 June 2021/Published online: 8 October 2021

(c) Dr. K C Chaudhuri Foundation 2021

\begin{abstract}
Objective To determine epilepsy and neurodevelopmental outcomes beyond $2 \mathrm{y}$ of age and their putative prognostic factors in children with West syndrome (WS).

Methods This cross-sectional study was initiated after approval from Institutional Ethics Committee. A follow-up cohort of 114 children (aged $\geq 2 \mathrm{y}$ ) diagnosed and treated for WS at the authors' center were assessed in-person for epilepsy and neurodevelopmental outcomes using Vineland Social Maturity Scale - Malin's adaptation for Indian children. Subsequently, age at onset, lead-time-to-treatment, etiology, and response to any of the standard therapies were analyzed as possible predictors of these outcomes.

Results Of 114 children (mean age: $55 \pm 32$ mo, 91 boys), structural etiology was the predominant underlying etiology (79.8\%) for WS. At 2 y of age, $64 \%$ had ongoing seizures. At the last follow-up, $76 \%$ had social quotient $<55$, and $39 \%$ had cerebral palsy (spastic quadriparesis in 21\%). An underlying structural etiology was associated with ongoing seizures [OR (95\% CI) 3.5 (1.4-9); $p=0.008$ ] at 2 y of age and poor developmental outcomes [OR (95\% CI): $3.3(1.3-8.9) ; p=0.016$ ]. Complete cessation of spasms with the standard therapy was significantly associated with better seizure control [OR (95\% CI): 5.4 (2.3-13); $p<0.001]$ and neurodevelopmental outcome [OR (95\% CI): $5.2(1.8-14.9) ; p<0.001$ ].

Conclusion The majority of children with WS have a poor neurodevelopmental outcome and epilepsy control on follow-up. The underlying etiology and response to initial standard therapy for epileptic spasms have a prognostic role in predicting the neurological outcome in these patients on follow-up.
\end{abstract}

Keywords West syndrome $\cdot$ Infantile spasms; Long-term outcome $\cdot$ Children $\cdot$ Epilepsy $\cdot$ Developmental outcome

\section{Introduction}

West syndrome (WS) is a rare age-dependent epilepsy syndrome characterized by epileptic spasms (ES) in clusters, hypsarrhythmia on interictal electroencephalography (EEG), and is often associated with developmental delay or

Pratibha Singhi

doctorpratibhasinghi@gmail.com

1 Department of Pediatrics, Postgraduate Institute of Medical Education and Research (PGIMER), Chandigarh, India

2 Pediatric Neurology Division, Department of Pediatrics, PGIMER, Chandigarh, India

3 Child Psychology Division, Department of Pediatrics, PGIMER, Chandigarh, India

4 Pediatric Neurology and Neurodevelopment, Medanta, The Medicity, Gurgaon, Haryana 122001, India regression [1]. It is usually associated with a poor neurocognitive outcome, with early death (in one-third of patients) or frequent progression into other epilepsy syndromes [2-6]. The children with unknown etiology (previously referred to as cryptogenic ones) usually fare better in seizure and cognitive outcomes [2,3].

There is a relative paucity of literature on epilepsy and neurodevelopmental outcomes in Indian children with WS. Most studies from developed countries report outcomes at different time-points ranging from 18 mo of therapy to adulthood [4]. These studies reveal that approximately half to two-thirds of the surviving children have persistent seizures, with $18 \%-23 \%$ progressing to Lennox-Gastaut syndrome (LGS), and nearly three-fourths of them have significant developmental delay [2, 4, 7-10]. However, most of these studies provided a subjective estimate of outcomes and did not look at the electroencephalographic outcome on followup. The key predictors of poor outcomes identified in these 
studies include structural etiology, early age at onset $(<4$ mo), presence of developmental delay before the onset of ES, longer lead time to treatment (LTTT), and no response to standard therapy [adrenocorticotrophic hormone (ACTH) in most studies] [2, 4, 7-10]. Few studies identified the presence of adverse perinatal events and female gender as poor prognostic factors [11].

Considering the preponderance of structural etiology and longer LTTT for WS in the developing world, epilepsy and neurodevelopmental outcomes in WS are presumed to be dismal here [12, 13]. Most of the literature from India targets the clinical profile of WS and therapies for epileptic spasms, with very few studies assessing epilepsy and neurodevelopmental outcome [14-21]. The current study aimed to objectively evaluate epilepsy and neurodevelopmental outcome in a follow-up cohort with WS.

\section{Material and Methods}

The current cross-sectional study was conducted over $1 \mathrm{y}$ (January-December 2013) at a tertiary-care pediatric hospital in Northern India after approval from Institutional Ethics Committee. Written informed consent was obtained from guardians of all the enrolled children.

The primary objective was to determine the seizure control (at 2 y chronological age) and neurodevelopmental status at the last follow-up for children with WS. The secondary objective was to determine the putative factors at baseline that predict poor epilepsy and neurodevelopmental outcome.

A follow-up cohort of the children (aged $\geq 2 \mathrm{y}$ ) diagnosed and treated for WS at the authors' center were enrolled after informed consent. Those with a minimum of three follow-up visits after starting treatment were enrolled. All the enrolled children had a baseline EEG documenting the presence of hypsarrhythmia (classical or modified) before the initiation of therapy. Those with incomplete baseline data were excluded.

Baseline details including demographic details, disease and treatment details such as age at onset, LTTT, etiology, findings on investigations (neuroimaging and EEG), nature of therapies received, and relapses, if any, were recorded using a prestructured case record form (based on history, examination, and clinic records).

Response to standard therapies in the form of complete cessation of ES was assessed. For the current study, complete cessation was defined as no clinical spasms witnessed within $14 \mathrm{~d}$ of commencement of standard therapy and cessation lasting for $\geq 28$ consecutive days from the time of the last witnessed spasms. After complete cessation, relapse was defined as (a) any episode of spasms occurring in clusters; (b) two or more episodes of spasms that occur singly but not in clusters; and (c) subtle spasms (if accompanied by an EEG showing hypsarrhythmia).
Seizure outcomes at a chronological age of 2 y were categorized into seizure freedom (for $\geq 3 \mathrm{mo}$ ) and continuing seizures. A follow-up EEG obtained at $\geq 2$ y of age was assessed for any abnormalities. A diagnosis of LGS was made by a pediatric neurologist based on the clinical and EEG criteria.

Children were assessed in-person for neurodevelopmental outcomes. Malin's adaptation of the Vineland Social Maturity Scale (VSMS) was performed to assess the social quotient (SQ) [22]. This particular scale was used considering the wide age range of the enrolled study population. VSMS comprises 89 items with a score ranging from 1 to 140 . Since a SQ of 55 or more indicates that a child is trainable to do his activities of daily living, a cutoff of 55 was taken to indicate a poor versus fair outcome [23]. VSMS scores were subcategorized into the following categories: $\geq 70,55-70,40-55,25-40$, and $<25$.

Age at onset, lead-time-to-treatment, etiology, and response to any of standard therapy were analyzed as possible predictors of the epilepsy and neurodevelopmental outcomes.

The data were collected on a structured case-record form. The data procured from the file review were coded and entered in statistical package for social sciences (SPSS) version 20 for Windows (SPSS, IBM) for analysis. In the descriptive analysis, the mean (with standard deviation) and frequencies (with percentage) were computed for continuous variables and categorical variables, respectively. Binary associations were evaluated using the chi-square test and Fisher exact test where applicable. A $p$ value of less than 0.05 was considered statistically significant unless otherwise specified. Odds ratios were calculated for the various predictor variables.

\section{Results}

A total of 309 children diagnosed with WS and treated at the authors' centre from January 2005 to December 2012 were screened for the study's eligibility. Of 309 children, 195 were excluded ( 13 children expired, 5 children declined consent, and 177 were lost to follow-up). A total of 114 patients were enrolled in the study. Of the enrolled children, the follow-up duration varied as: $<2$ y ( 6 children), $2-5$ y (92 children), and $>5$ y ( 16 children).

The mean age at enrollment was $55 \pm 32$ mo (range: 24-114) with a male to female ratio of $4: 1$. The mean age at onset of spasms was $6.3 \pm 4.6$ mo. The mean LTTT (defined as the time from onset of spasms to initiation of either hormonal therapy or vigabatrin) was $9.4 \pm 10.6$ mo (range: 0.2-61). Among the study subjects, 83 (73\%) children had microcephaly, and 96 children $(84 \%)$ had a developmental delay at baseline, i.e., before the onset of 
Table 1 Baseline characteristics of the cohort

\begin{tabular}{ll}
\hline Baseline characteristics & $N=114$ \\
\hline Mean age at enrollment in months \pm SD (range) & $55 \pm 32$ \\
Male gender; $N(\%)$ & $91(79.8)$ \\
Pre-existing seizures of other semiology; $N(\%)$ & $57(50)$ \\
Pre-existing developmental delay; $N(\%)$ & $96(84)$ \\
Mean age at onset of spasms in months \pm SD (range) & $6.3 \pm 4.6$ \\
Age at onset of spasms $\leq 4$ mo & (range: $0.1-26.0)$ \\
Mean treatment lag in months \pm SD (range) & $42(37 \%)$ \\
Etiology; $N$ (\%) & $9.4 \pm 10.6$ mo \\
Structural & $($ range: $0.2-61)$ \\
Malformations & \\
Postnatally acquired brain insult & $91(79.8)$ \\
Hypoxic brain injury & $5(4.4)$ \\
Hypoglycemic brain injury & $73(64)$ \\
Perinatal stroke sequelae & $49(43)$ \\
Postmeningitic sequelae & $20(17.5)$ \\
Others (congenital hydrocephalus, congenital infections) & $2(1.7)$ \\
Nonstructural (presumed genetic) & $2(1.7)$ \\
\hline
\end{tabular}

spasms. Ninety-one children $(79.8 \%)$ had structural brain lesions due to various known etiology (Table 1).

Of the enrolled children, 61 (53.5\%) had complete cessation of spasms (lasting for $\geq 28 \mathrm{~d}$ ) within $14 \mathrm{~d}$ of initiation of first-line therapy, while the rest had no response, as illustrated in Table 2. Of 61 responders, nearly two-thirds of children (39) had relapsed on follow-up.

At the chronological age of $2 \mathrm{y}, 42(36 \%)$ of the enrolled children were seizure-free, while $72(64 \%)$ had persistent seizures. The follow-up EEG was available in 103 children (age range 2-8 y). It was abnormal in 80 children $(78 \%)$, with 17 (15\%) children showing evolution to Lennox-Gastaut syndrome. Eighty-two children (72\%) were on polypharmacy with three or more antiepileptic drugs needed to control seizures. Response to standard therapy [Odds ratio (OR; 95\% CI): $5.4(2.3-13) ; p<0.001]$ and nonstructural etiology [OR (95\% CI) 3.5 (1.4-9); $p=0.008$ ] were associated with better seizure control at 2 y of age (Table 3 ).

On follow-up, 87 (76\%) of the 114 children were not trainable with a social quotient $<55$ (Table 2). Of the 114 patients, $45(39 \%)$ had cerebral palsy, with $24(21 \%)$ of them having spastic quadriparesis. Seventy-three (64\%) had other comorbidities identified on follow-up, like behavioral problems, autism, hyperactivity, hearing and vision deficits.

No response to initial standard therapy [OR $(95 \% \mathrm{CI})$ : 5.2 (1.8-14.9); $p<0.001]$, and structural etiology [OR (95\% CI): 3.3 (1.3-8.9); $p=0.016]$ were associated with poor neurodevelopmental outcome $(\mathrm{SQ}<55)$ (Table 4).

\section{Discussion}

The current study depicts the poor neurodevelopmental outcome in a follow-up cohort of Indian children with WS. There was substantial attrition (to the tune of $60 \%$ ) in the present study, reflecting the difficulties in long-term follow-up in resource-constrained settings. Attrition, an important aspect of WS research, is not depicted well in the previous follow-up studies from India, which evaluated children in the desired age group who continued to follow up (without mentioning the numbers of those diagnosed and treated at their center) [20, 21]. Significant loss to followup might be responsible for the lack of mortality data in these

Table 2 Epilepsy and neurodevelopmental outcomes in children with West syndrome

\begin{tabular}{lc}
\hline Outcomes & $N=114$ \\
\hline Clinical response to standard therapy; $N(\%)$ & \\
$\quad$ Complete cessation of spasms & $61(53.5)$ \\
$\quad$ No response & $53(46.5)$ \\
Evolution to Lennox-Gastaut syndrome; $N(\%)$ & $17(15 \%)$ \\
Seizure status at 2 y; $N(\%)$ & \\
$\quad$ Seizure freedom & $42(36)$ \\
Ongoing seizures & $72(64)$ \\
Social quotient as per VSMS; $N(\%)$ & \\
$\geq 70$ & $16(14)$ \\
$55-69$ & $11(9.6)$ \\
$40-54$ & $9(7.9)$ \\
$26-39$ & $19(16.7)$ \\
$\leq 25$ & $59(51.8)$ \\
\hline
\end{tabular}


Table 3 Effect of various risk factors for persistent seizures at $2 \mathrm{y}$ of age

\begin{tabular}{llll}
\hline Characteristics & $\begin{array}{l}\text { Continuing seizures } \\
(\%)\end{array}$ & Cessation (\%) & $p$ value \\
\hline Age at onset of spasms & & & \\
$\quad \leq 4$ mo & $28(38.9)$ & $14(33.3)$ & 0.55 \\
$\quad>4$ mo & $44(61.1)$ & $28(66.6)$ & \\
Lead time to treatment & $63(87.5)$ & $38(90.5)$ & 0.44 \\
$\quad>1$ mo & $9(12.5)$ & $4(9.5)$ & \\
$\leq 1$ mo & & & \\
Response to standard therapy* & $43(60)$ & $9(22)$ & OR (95\% CI) $5.4(2.3-13)$ \\
$\quad$ No response & $29(40)$ & $33(78)$ & \\
$\quad$ Complete cessation & $63(87.5)$ & $28(66.7)$ & 0.008 \\
Etiology & $9(12.5)$ & $14(33.3)$ & OR (95\% CI) $3.5(1.4-9)$ \\
$\quad$ Structural & & & \\
$\quad$ Nonstructural &
\end{tabular}

*Response to standard therapy was defined as clinical cessation of spasms with $14 \mathrm{~d}$ of initiation of standard therapy, lasting for $\geq 28 \mathrm{~d}$

CI Confidence interval, $O R$ Odds ratio cross-sectional studies [20, 21]. The dearth of prospective longterm follow-up and natural history studies on WS in India might also be accountable for the nonexistence of reliable mortality statistics for WS from India.

The baseline characteristics of the cohort (age at onset, long LTTT, a preponderance of male gender, and structural etiology) were comparable with the previous literature from India [12]. Most of the enrolled children belonged to the $2-5$ y age group. Of the children who could be contacted for study (132/309), mortality was nearly $10 \%$ (13/132). This might not be a truly representative figure (considering the sizeable attrition), but this is comparable with the Western literature (11\%-13\% mortality before the age of 3 y) [4].

Nearly half of the enrolled children had a complete cessation of spasms after any of the standard therapy. The response rate was higher than the response rate to hormonal treatment in South Asia [12]. This might probably be accounted for by attrition and the fact that EEG resolution of hypsarrhythmia was not a response criterion in the current study. At the age of two, one-third of the enrolled children had attained seizure freedom for at least 3 mo. This was lower than that reported in the other two Indian cohorts $(61 \%$ and $47 \%$ ) since these studies analyzed epilepsy outcomes at the last follow-up (instead of a fixed age of $2 \mathrm{y}$ as done in the current study) [20, 21]. Similar to Western literature, structural etiology and response to standard therapy were significant predictors of epilepsy outcome [24, 25]. In the study by Sehgal et al., LTTT $<3$ mo and response to first-line therapy were found to be associated with a favorable outcome [20]. However, none of these factors was found to be associated with epilepsy outcomes in the study by Gupta et al. [21]. LTTT was not a significant predictor in the present study
Table 4 Predictors of neurodevelopmental outcomes in children with West syndrome aged more than 2 y (social quotient based on Vineland Social Maturity Scale)

\begin{tabular}{|c|c|c|c|}
\hline Characteristics & $\mathrm{SQ}>55(\%)$ & $\mathrm{SQ}<55(\%)$ & $p$ value \\
\hline \multicolumn{4}{|l|}{ Age at onset of spasms } \\
\hline$\leq 4 \mathrm{mo}$ & $6(22)$ & $36(41)$ & 0.055 \\
\hline$>4 \mathrm{mo}$ & $21(78)$ & $51(59)$ & OR $(95 \%$ CI $): 2.5(0.9-6.7)$ \\
\hline \multicolumn{4}{|l|}{ Treatment lag } \\
\hline$>1 \mathrm{mo}$ & $24(88.9)$ & $77(88.5)$ & 0.63 \\
\hline$\leq 1 \mathrm{mo}$ & $3(11.1)$ & $10(11.5)$ & \\
\hline \multicolumn{4}{|c|}{ Response to standard therapy* } \\
\hline No response & $5(19)$ & $47(54)$ & $<0.001$ \\
\hline Complete cessation & $22(81)$ & $40(46)$ & OR $(95 \% \mathrm{CI}): 5.2(1.8-14.9)$ \\
\hline \multicolumn{4}{|l|}{ Etiology } \\
\hline Structural & $17(63)$ & $74(85)$ & 0.016 \\
\hline Nonstructural & $10(37)$ & $13(15)$ & OR (95\% CI): $3.3(1.3-8.9)$ \\
\hline
\end{tabular}

*Response to standard therapy was defined as clinical cessation of spasms with $14 \mathrm{~d}$ of initiation of standard therapy, lasting for $\geq 28 \mathrm{~d}$

$C I$ Confidence interval, $O R$ Odds ratio, $S Q$ Social quotient 
since there were very few children in the group with acceptable LTTT ( $\leq 1 \mathrm{mo})$.

In the current study, approximately three-fourths of enrolled children had an SQ $<55$ and were not trainable. This was similar to the Finnish study [9]. The two Indian studies used a different psychometric tool (Developmental Profile 3) for assessment with unfavorable outcome (general development score $<70$ ) seen in nearly $90 \%$ of children, which was comparable with an SQ $<70$ in $86 \%$ of children in the current study $[20,21]$. Like previous studies, structural etiology and response to standard therapy were significant predictors of neurodevelopmental outcome in the present study $[9,21,24]$. None of the analyzed factors were found to affect the neurodevelopmental outcomes in the study by Sehgal et al. [20]. Although LTTT has been identified as an important predictor of neurodevelopmental outcome in various studies, the association was not significant in the present study, probably due to the reasons stated previously $[3,9,21]$. Also, there was a trend towards significance for early age at onset as a neurodevelopmental outcome predictor in the present study similar to the Finnish cohort [9].

The present study gives a real-world reflection of difficulties (specifically attrition) in long-term research on WS in developing countries. Teleneurology can be a solution to some of these difficulties and might help maintain a robust follow-up. The COVID-19 pandemic should be taken as an opportunity for infrastructure building for telehealth services, which can later be used for rigorous follow-up [26, 27]. Besides, the current study performed an objective assessment of neurodevelopmental outcomes using a validated scale in a large cohort of children with WS. Although dedicated screening and diagnostic evaluation for comorbid neurobehavioral disorders such as autism spectrum disorders could have been helpful, this could not be done. The reasons for attrition were not clear since the patients were not contactable.

\section{Conclusions}

WS is associated with poor epilepsy and neurodevelopmental outcomes on follow-up. The underlying etiology and response to the standard therapy are significant predictors of epilepsy and neurodevelopmental outcomes. Further large and prospective cohort studies are needed to assess the long-term outcomes and mortality in Indian children with WS.

Authors' Contributions RA, JKS, Prahbhjot M, NS, and PS were involved in study design, data interpretation, data analysis, and critical review of the manuscript. RA was also involved in data collection.
Priyanka $\mathrm{M}$ was involved in data interpretation, data analysis, and critical review of the manuscript. PS is the guarantor for this paper.

\section{Declarations}

Conflict of Interest None.

\section{References}

1. Lux AL, Osborne JP. A proposal for case definitions and outcome measures in studies of infantile spasms and west syndrome: consensus statement of the west delphi group. Epilepsia. 2004;45:1416-28.

2. Vendrame M, Guilhoto LMFF, Loddenkemper T, Gregas M, Bourgeois BF, Kothare SV. Outcomes of epileptic spasms in patients aged less than 3 years: single-center United States experience. Pediatr Neurol. 2012;46:276-80.

3. Widjaja E, Go C, McCoy B, Snead OC. Neurodevelopmental outcome of infantile spasms: a systematic review and metaanalysis. Epilepsy Res. 2015;109:155-62.

4. Riikonen R. Infantile Spasms: Outcome in clinical studies. Pediatr Neurol. 2020;108:54-64.

5. Harini C, Nagarajan E, Bergin AM, et al. Mortality in infantile spasms: a hospital-based study. Epilepsia. 2020;61:702-13.

6. Sillanpää M, Riikonen R, Saarinen MM, Schmidt D. Longterm mortality of patients with west syndrome. Epilepsia Open. 2016;1:61-6.

7. Lagae L, Verhelst H, Ceulemans B, et al. Treatment and longterm outcome in west syndrome: the clinical reality. a multicentre follow up study. Seizure. 2010;19:159-64.

8. Kivity S, Lerman P, Ariel R, Danziger Y, Mimouni M, Shinnar $\mathrm{S}$. Long-term cognitive outcomes of a cohort of children with cryptogenic infantile spasms treated with high-dose adrenocorticotropic hormone. Epilepsia. 2004;45:255-62.

9. Riikonen R. Long-term otucome of west syndrome: a study of adults with a history of infantile spasms. Epilepsia. 1996;37:367-72.

10. O'Callaghan FJ, Lux AL, Darke K, et al. The effect of lead time to treatment and of age of onset on developmental outcome at 4 years in infantile spasms: evidence from the United Kingdom Infantile Spasms Study. Epilepsia. 2011;52:1359-64.

11. Matsumoto A, Watanabe K, Negoro T, et al. Long-term prognosis after infantile spasms: a statistical study of prognostic factors in 200 cases. Dev Med Child Neurol. 1981;23:51-65.

12. Madaan P, Chand P, Linn K, et al. Management practices for west syndrome in South Asia: a survey study and meta-analysis. Epilepsia Open. 2020;5:461-74.

13. Vaddi VK, Sahu JK, Dhawan SR, Suthar R, Sankhyan N. Knowledge, attitude and practice (KAP) study of pediatricians on infantile spasms. Indian J Pediatr. 2018;85:836-40.

14. Singhi P, Ray M. Profile of west syndrome in North Indian children. Brain Dev. 2005;27:135-40.

15. Gulati S, Jain P, Kannan L, Sehgal R, Chakrabarty B. The clinical characteristics and treatment response in children with west Syndrome in a developing country: a retrospective case record analysis. J Child Neurol. 2015;30:1440-7.

16. Sahu JK, Madaan P. What matters the most for hormonal therapy in west syndrome: drug, dosage, or duration? Indian J Pediatr. $2021 ; 88: 751-2$.

17. Chandra S, Bhave A, Bhargava R, Kumar C, Kumar R. West syndrome: response to valproate. Front Neurol. 2012;3:166.

18. Angappan D, Sahu JK, Malhi P, Singhi P. Safety, tolerability, and effectiveness of oral zonisamide therapy in comparison with 
intramuscular adrenocorticotropic hormone therapy in infants with west syndrome. Eur J Paediatr Neurol. 2019;23:136-42.

19. Rajpurohit M, Gupta A, Madaan P, Sahu JK, Singhi P. Safety, feasibility and effectiveness of pulse methylprednisolone therapy in comparison with intramuscular adrenocorticotropic hormone in children with west syndrome. Indian J Pediatr. 2021;88:663-7.

20. Sehgal R, Gulati S, Sapra S, Tripathi M, Kabra M, Pandey RM. Neurodevelopmental and epilepsy outcome in children aged one to five years with infantile spasms-a North Indian cohort. Epilepsy Res. 2014;108:526-34.

21. Gupta J, Sharma S, Mukherjee SB, Jain P, Aneja S. Neurodevelopmental and epilepsy outcomes of children with west syndrome: a cross-sectional study from North India. Ann Indian Acad Neurol. 2020;23:177-81.

22. Malin AJ. Vineland Social Maturity Scale-Nagpur Adaptation. Lucknow, India: Indian Psychological Corporation; 1971.

23. Singh S, Pandey S, Agarwal V. A comparative study of vineland adaptive behavior scale II and vineland social maturity scale on children and adolescents with intellectual disability. J. Ind. Assoc. Child Adolescent Mental Health. 2019;15:27-38.

24. Partikian A, Mitchell WG. Neurodevelopmental and epilepsy outcomes in a North American cohort of patients with infantile spasms. J Child Neurol. 2010;25:423-8.

25. Güveli BT, Çokar “O, D” ortcan N, Benbir G, Demirbilek V, Dervent A. Longterm outcomes in patients with west syndrome: an outpatient clinical study. Seizure. 2015;25:68-71.

26. Madaan P, Sahu JK, Wanigasinghe J, et al. Teleneurology based management of infantile spasms during COVID-19 pandemic: a consensus report by the South Asia allied west syndrome research group. Epilepsy Behav Rep. 2021;15:100423.

27. Sahu JK, Madaan P, Chand P, et al. Management of west syndrome during COVID-19 pandemic: a viewpoint from South Asian west syndrome research group. Epilepsy Res. 2020;167:106453.

Publisher's Note Springer Nature remains neutral with regard to jurisdictional claims in published maps and institutional affiliations. 